See discussions, stats, and author profiles for this publication at: https://www.researchgate.net/publication/336205739

\title{
Structural Reliability of Masonry Arch Bridges Subject to Natural Aging
}

Chapter · January 2020

DOI: 10.1007/978-3-030-29227-0_91

5 authors, including:

ค. Gianluca Borgna

University of Padova

1 PUBLICATION O CITATIONS

SEE PROFILE

Lorenzo Hofer

University of Padova

63 PUBLICATIONS 185 CITATIONS

SEE PROFILE
Mariano Angelo Zanini

University of Padova

120 PUBLICATIONS 773 CITATIONS

SEE PROFILE

Flora Faleschini

University of Padova

121 PUBLICATIONS 864 CITATIONS

SEE PROFILE

Some of the authors of this publication are also working on these related projects:

COST Action TU 1406 View project

Project fib Task Group 2.5 "Bond and Material Models" View project 


\title{
Structural Reliability of Masonry Arch Bridges Subject to Natural Aging
}

\author{
Gianluca Borgna ${ }^{1}$, Mariano Angelo Zanini ${ }^{1(凶)}$, Lorenzo Hofer ${ }^{1}$, \\ Flora Faleschini ${ }^{1}$, and Jose Matos ${ }^{2}$ \\ ${ }^{1}$ University of Padova, Via Marzolo 9, 35131 Padua, Italy \\ marianoangelo.zanini@dicea.unipd.it \\ ${ }^{2}$ University of Minho, Campus de Azurém, 4800-058 Guimarães, Portugal
}

\begin{abstract}
This paper deals with a simplified full-probabilistic methodology for the safety assessment of existing masonry arch bridges. The proposed framework aims to determine the ultimate load-carrying capacity (Ultimate Limit State) of a bridge subject to environmental deterioration, and to establish the influence on the structural reliability.
\end{abstract}

Keywords: Arch bridges - Railway bridges - Structural reliability · Deterioration process $\cdot$ Uncertainty

\section{Introduction}

According to the international union of railways (UIC - Union internationale des chemins de fer), masonry arch bridges are more than 200000 and represent almost $50 \%$ of the European total bridge stock [1]. Most of the them were built in the second half of the $19^{\text {th }}$ century and at the very beginning of the $20^{\text {th }}$ century. Usually a masonry arch bridges presents a well-defined structure in which some typical elements are present. In particular, under the structural point of view, five main elements that compose the mechanical behavior of the bridge can be individuated, as showed in Fig. 1.
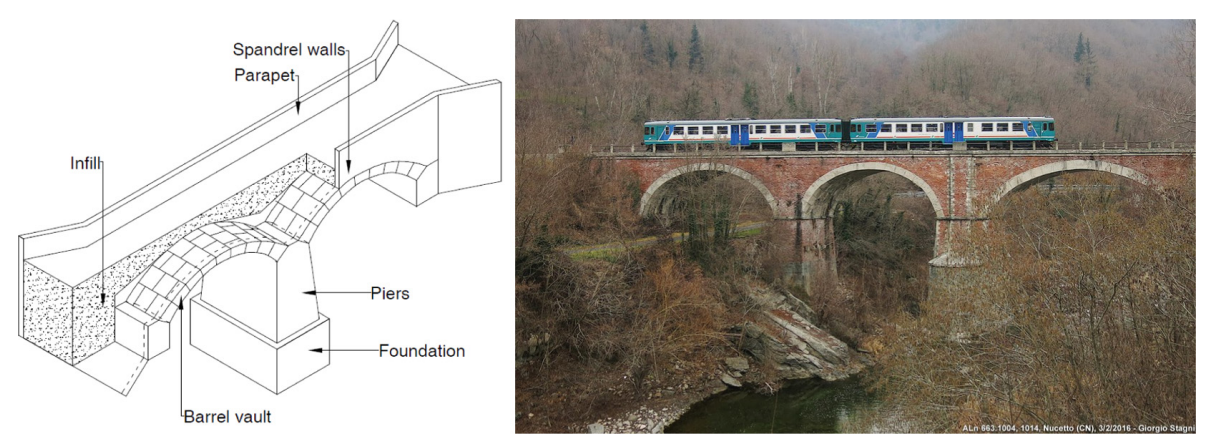

Fig. 1. (a) Main elements and (b) example of an existing masonry arch bridge (CN - Italy). 
Barrel vaults are the main structural element and provide the load bearing capacity of the bridge. Piers are then needed for transferring loads from vaults to the foundations. Over the barrel vaults, the infill is located with the following aims:

- level the transit area and the road surface;

- providing confinement to the barrel vaults allowing them to develop their structural strength;

- redistribute the point load produced by external actions.

Finally, spandrel walls are needed for providing lateral confinement to the infill. Even if some common structural elements can be individuated within this bridge typology, every bridge has its specific history, characteristics and maintenance condition. Although some bridges are in very good conditions, others need intermediate repairs, and most of the them are in between. The structural safety of every bridge depends on several factors that introduce in the safety assessment procedure many uncertainty sources.

The main concern is about the fact that old bridges are called to bear new traffic loads under a progressive materials' deterioration whose quantification many times is highly uncertain. Furthermore, the aged bridges may have sustained mechanical damages during their life introducing further uncertainty sources. For the need of knowing the bridges status, structural monitoring techniques have thus been developed, and are widely validated by years of use and refinement. Anyway, although they are a well-established practice in the management of infrastructure assets, they cannot be considered completely suitable and comprehensive. As a matter of fact, they are limited to the quality of the inspection, to the accuracy of the laboratory tests and to the other assessment methods. Furthermore, laboratory tests are usually limited to some specific points of the structure, proving high quality data only for those points of interest. It is not uncommon, to have very scattered data, implying the need to consider material properties as sources of uncertainties in the computation of the structural reliability. Loads, to which the bridge can be subject during its life-time, and their spatial distributions, represent other sources of uncertainties and need to be considered as random variables. In the specific case of masonry arch bridges, their load bearing capacity rely mainly on the arch ring and on the infill. The way in which these two components contribute to the arch's strength depends on the specific case. This particularity makes this type of structure even more subject to parameters uncertainty, thus introducing a significantly higher degree of variability, than respect to other types of bridges [2]. For these reasons, masonry arch bridges need probabilistic methods for a complete understanding of their structural safety. This paper wants to verify the reliability of a masonry arch bridge subject to environmental deterioration, modelled as a reduction of the mortar joints, and evaluate the influence of deterioration both on the structural reliability and on the load bearing capacity.

\subsection{Structural Reliability}

As previously introduced, risks in bridge assessment are posed by unreliability in several variables. Each of these variables can be defined using suitable probability distribution functions. A structure must fulfil the assigned functions, whether in the 
design of new structures or in safety assessment of existing ones. One of the most important indicators that measures the compliance of a structure with the purpose to which it was designed is the reliability index, usually indicated with $\beta$. In this paper the safety assessment is performed through the comparison between the structural resistance $R$ and applied loads $L$. The applied loads $L$ acting on the generic element and its resistance $R$ are modelled as random variables. At this point, the definition of a suitable limit state LS is needed. Formally the exceedance of a limit state is defined by a LS function $g\left(X_{i}\right)$, that generally will be function of a series RVs $X_{i}$ involved in both the computation of capacity and demand. The LS function is thus defined as [3]:

$$
G\left(X_{i}\right)=R-L
$$

The verification in this case is of the type:

$$
G>0(\text { Safe })
$$

while failure will occur when:

$$
G<0 \text { (Failure) }
$$

The objective of the reliability analysis, is the computation of the failure probability $P_{f}$, defined as

$$
P_{f}=P[G(R, L) \leq 0]=P[R \leq S]
$$

In many cases, the computation of Eq. (4) can not be immediate; furthermore, $P_{f}$ is in the order of $10^{-4}-10^{-5}$, and numerically some issues may arise when dealing with its estimation. For this scope, it is convenient to introduce the so-called reliability index $\beta$ that is connected to the probability of failure with the following equation

$$
\beta=-\Phi^{-1}\left(P_{f}\right)
$$

In which the operator $\Phi$ is cumulative density function (CDF) of the standard normal distribution. In the simplest case, the reliability index can be computed from the first two moments of the load and resistance distribution in the following way [3]:

$$
\beta=\frac{\mu_{R}-\mu_{L}}{\sqrt{\sigma_{R}^{2}+\sigma_{L}^{2}}}
$$

where

$$
\begin{aligned}
& \mu_{R}: \text { average value of resistance } \\
& \mu_{L}: \text { average value of load } \\
& \sigma_{R}: \text { standard deviation of resistance } \\
& \sigma_{L}: \text { standard deviation of load }
\end{aligned}
$$


Once computed $\beta, P_{f}$ can be derived from Eq. (5) as

$$
P_{f}=\Phi(-\beta)
$$

\section{Railway Actions}

The Load Model 71 defined in the "Eurocode 1: Action on Structures" has been adopted for representing the normal rail traffic [4]. In particular, the load model LM71 presents as characteristic value of the concentrated loads $250 \mathrm{kN}$ and the value of 80 $\mathrm{kN} / \mathrm{m}$ for the distributed load. Moving loads, such as railway loads, cause dynamic effects on the structure, resulting in the amplification of the entire structural behavior. For this reason, current norm [4] adopts dynamic factors for amplifying the static load and taking into account their dynamic nature. Generally, the dynamic factor is taken as either $\phi_{2}$ or $\phi_{3}$ depending on the quality of track maintenance.

For carefully maintained track [4]:

$$
\phi_{2}=\frac{1,44}{\sqrt{L_{\phi}}-0,2}+0,82
$$

For standard maintained track [4]:

$$
\phi_{3}=\frac{2,16}{\sqrt{L_{\phi}}-0,2}+0,73
$$

where $L_{\phi}$ is the determinant length, i.e. the span length $L$ for simply supported bridges, or $L_{\phi}$ for other type of bridges. For arch bridges, according to EN 1991-2 [4], the determinant length $L_{\phi}$ is half of the clear opening for single span bridges, while it is twice the clear opening for a series of arches. In the case of arch bridges and concrete bridges of all types with a cover of more than $1,00 \mathrm{~m}, \phi_{2}$ and $\phi_{3}$ may be reduced as follows:

$$
\operatorname{red} \phi_{2,3}=\phi_{2,3}-\frac{h-1}{10}
$$

where $h$ represents the height of the cover, that includes the ballast from the top of the deck to the top of the sleeper given (in meters) [4].

\section{Case Study}

For the purposes of the study, an Italian railway bridge (Cevetta bridge, CN) was selected and modelled in order to compute the reliability index as a function of degradation scenarios. Information on the bridge regarding the main geometrical properties (such as span, ring thickness, rise, arch width, maximum pier height, pier top width, number of spans, number of track lines) have been directly derived from the study [6]. 
In order to obtain the ultimate load-carrying capacity of bridge under investigation, numerical models were developed in the software Limit State: RING [5]. Figure 2 shows the adopted model. As previously introduced, the failure load factor has been computed in reference to the railway load model LM71, in which the four concentrated mobile live loads were considered acting progressively along the bridge length. For each position, the software calculates the kinematic collapse and the load multiplier. Regarding the load transmission mechanism, the masonry arch width in the transversal direction that resists the applied loading, i.e., maximum effective bridge width, is assumed equal to the width of the bridges with a transverse angle of distribution through ballast of $15^{\circ}$ and a transverse angle of distribution through fill of $30^{\circ}$ [5]. The passive pressure, provided by the fill, is obtained through the Rankine theory.

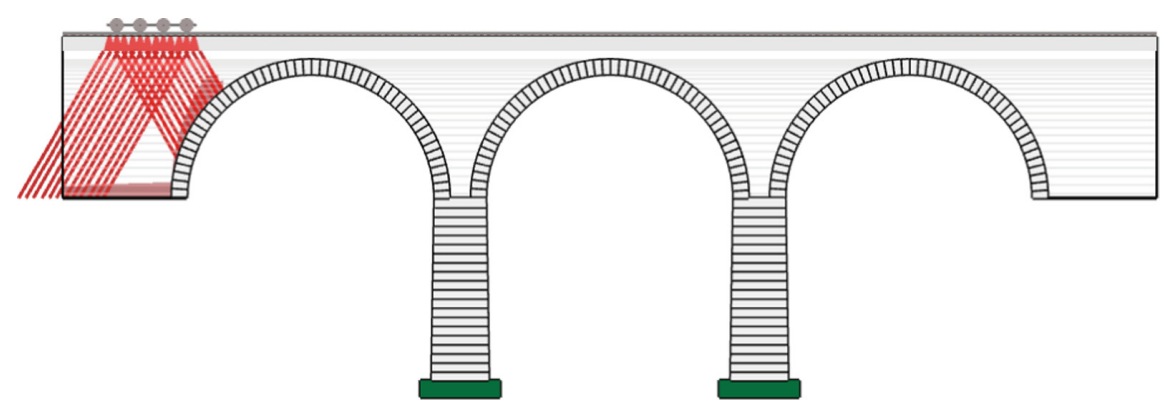

Fig. 2. Cevetta bridge $(r / s=0.5)$

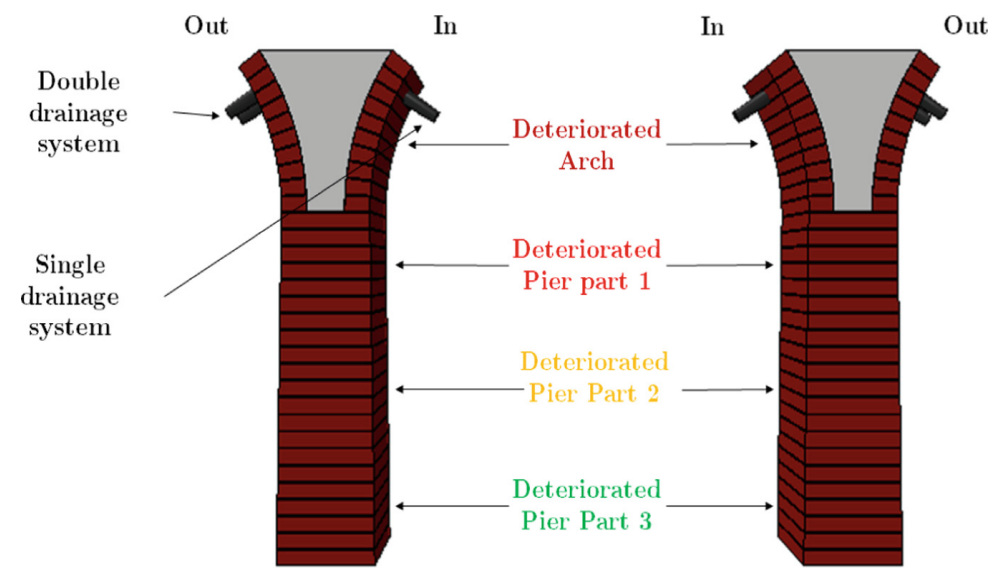

Fig. 3. Cevetta drainage system

Since passive pressure is fully mobilized only for high deformations and displacements of sections of an arch into surrounding fill, according to [6] a reduction factor of $50 \%$ was applied to passive pressure, in order to consider this fact. In the longitudinal direction, the live load dispersion is performed according to the 
Boussinesq theory, with a longitudinal angle of distribution of $15^{\circ}$ and $30^{\circ}$, through ballast and fill material respectively. For computing the resistance distribution, ten random variables have been assumed (masonry specific weight, compressive strength, friction coefficient, soil specific weight, soil friction angle, soil cohesion, ballast specific weight and height, crown depth and backfill height). Monte-Carlo method has thus been used for sampling 100 random vectors with whom perform structural analysis. Resistance values, one for each random vector, have thus been fitted for obtaining the resistance distribution. Regarding the load distribution, the average value of load model LM71 was normalized in order to correspond to a unitary failure load factor and a Coefficient of Variation $(\mathrm{CoV})$ of $10 \%$ was adopted. This value is then affected by the dynamic amplification coefficient $\phi_{3}$ and the reduced dynamic factor $\phi_{2,3}^{\text {red }}$ proposed by the European standard [4]. The deterioration of the arch bridges has been modeled by applying mortar losses along the intrados and extrados [5]. Accordingly, some degradation scenarios were assumed. Cevetta bridge is a 3-span masonry arch bridge with a rise to span ratio of 0.5 . It has been modeled with 50 blocks for every arch, while 21 blocks have been adopted for the piers, corresponding respectively to 50 and 21 contacts. For this bridge, a rainfall drainage system inside the arch and above the piers has been assumed (Fig. 3). In particular, there are 2 pipes outwards, while only 1 pipe inwards. The drainage system is assumed mirrored. The degradation scenarios hypothesized envisage the progressive reduction of the contact lengths generated both by the discharge of the water and by a possible breakage of the pipes.

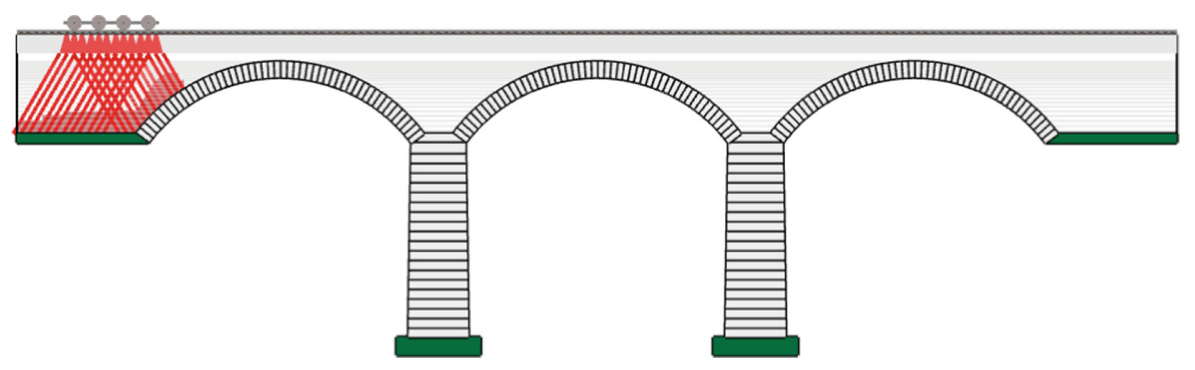

Fig. 4. Cevetta rise halved $(r / s=0.25)$

The part most subjected to degradation is the area of the arch interested by the drainage system to which a progressive reduction of mortar of 5\%,10\%, 15\%, 20\% was assumed. Concerning the piers, has been assumed a degradation of $0 \%, 5 \%$ and $10 \%$, $15 \%$, accordingly to the arch deterioration. In the hypothesis that the drainage system on one side is double outwards while single inwards, the degradation acting in the part affected by only one system is half that of the other. In particular, the adopted software allows modelling the reduction of mortar, by reducing the effective thickness of the arch barrel and pier [5]. Finally, for considering the influence of the geometry on the structural safety and its reduction due to progressive degradation, a different ratio of $r / s=0.25$ has been analyzed. Figure 4 shows the geometry of the Cevetta bridge with the ratio $\mathrm{r} / \mathrm{s}$ halved. Results show that, as the degradation manifests itself, the distribution of loadbearing capacity goes leftwards and slightly enlarges in the first case, whilst in the second remains constant, as depicted in Fig. 5. 


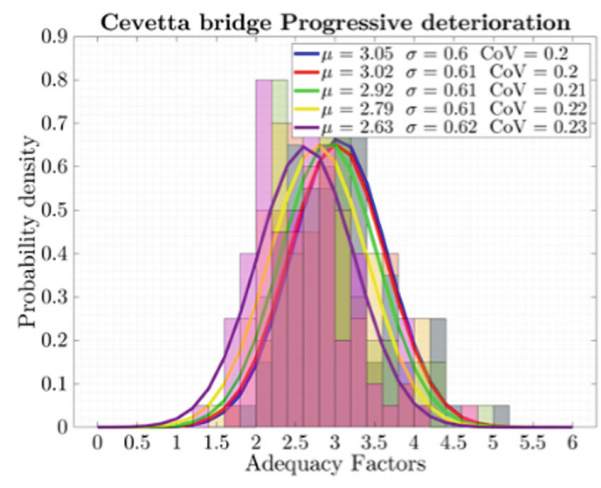

(a)

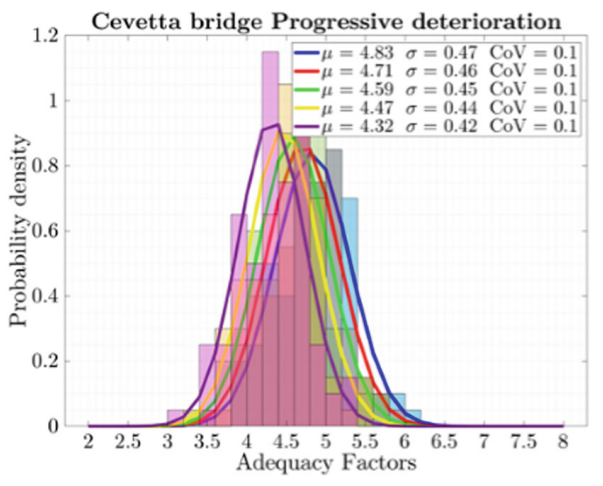

(b)

Fig. 5. Cevetta bridge resistance reduction - (a) $r / s=0.5-$ (b) $r / s=0.25$

Figure 6 shows the reduction of the reliability index for the five increasing degradation levels, and for both the geometrical conditions $r / s=0.5$ and $r / s=0.25$. Results show that the reliability index is strongly influenced by the $r / s$ ratio, while the maintenance level has a lower effect. In particular, the second bridge with $r / s=0.25$ has a reliability index that is more than two times the reliability index associated to the bridge with $r / s=0.50$. Finally, Fig. 7 shows how materials' deterioration affects the two bridges' structural reliability. In the first case $(r / s=0.5)$ the degradation of scenario 5 reduces of $25 \%$ the reliability index, while in the second case $(r / s=0.25)$ degradation phenomena slightly affect the bridge structural reliability (less than $5 \%$ ).
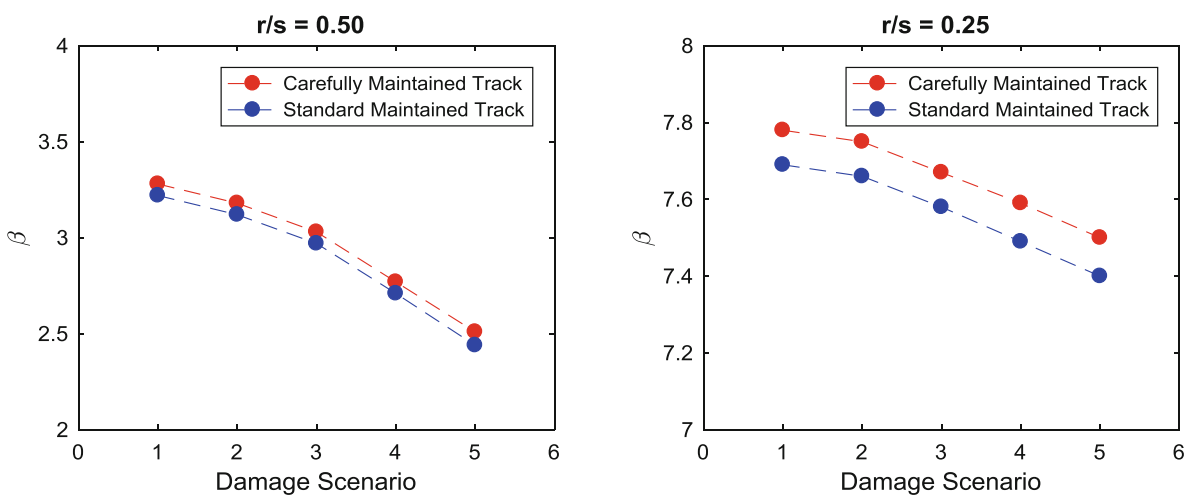

Fig. 6. Reliability reduction - (a) Cevetta bridge $(r / s=0.50)-$ (b) Cevetta bridge rise halved $(r / s=0.25)$ 

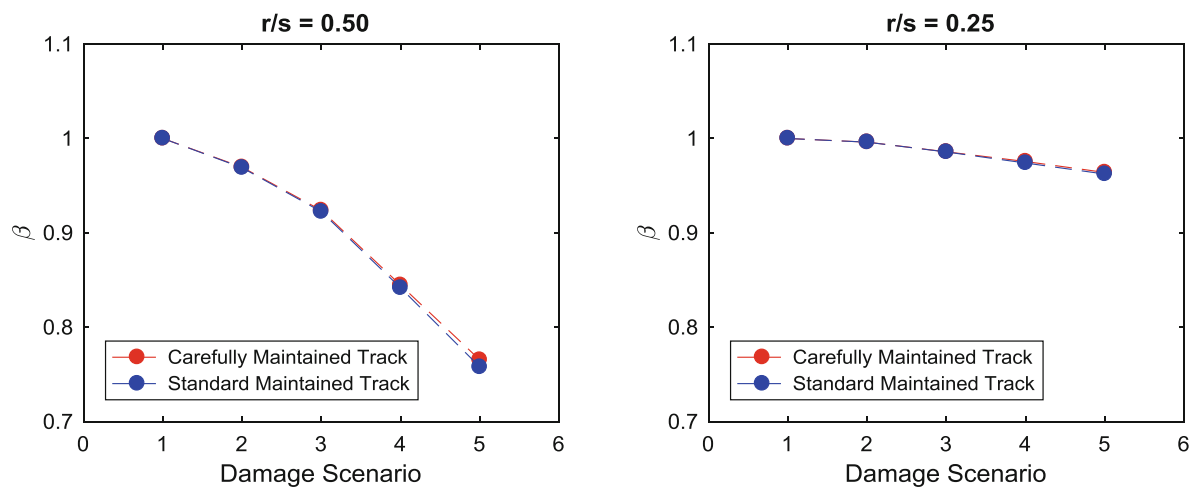

Fig. 7. Percentage reliability reduction - (a) Cevetta bridge $(r / s=0.50)-(b)$ Cevetta bridge rise halved $(r / s=0.25)$

\section{Conclusions}

This paper addressed the probabilistic safety assessment of a railway masonry arch bridge subject to environmental deterioration, showing the importance of considering degradation in structural reliability analysis. The $r / s$ ratio has a strong influence on both the structural reliability and on how it decreases for materials' deterioration processes. When Cevetta bridge is assumed with a halved $r / s(=0.25)$ ratio, the reliability index rose more than twice respect to the previous case. Environmental deterioration processes have a stronger influence, in terms of reliability index, on arch bridges with a high $r / s$ ratio, than respect to arch bridges with a low $r / s$ ratio.

\section{References}

1. UIC - Union internationale des chemins de fer. https://uic.org/track-structure. Accessed 4 June 2019

2. Halden, D.: Performance Criteria in Arch Bridge Assessment, Arch bridges. ICE Virtual Library (2015)

3. Nowak, A.S., Collins, K.R.: Reliability of Structures. CRC Press, New York (2012)

4. EN 1991-2 (2003) Eurocode 1: Actions on structures - Part 2: Traffic loads on bridges [Authority: The European Union Per Regulation 305/2011, Directive 98/34/EC, Directive 2004/18/EC], vol. 2, no. 2003 (2011)

5. LimitState : RING Manual, p. 44 (2016)

6. Moreira, V.N., Fernandes, J., Matos, J.C., Oliveira, D.V.: Reliability-based assessment of existing masonry arch railway bridges. Constr. Build. Mater. 115, 544-554 (2016) 